“ (C) 2017 IEEE. Personal use of this material is permitted. Permission from IEEE must be obtained for all other uses, in any current or future media, including

reprinting/republishing this material for advertising or promotional purposes, creating new collective works, for resale or redistribution to servers or lists, or reuse of any copyrighted component of this work in other works." 


\title{
A Bandwidth-Enhanced, Compact, Single-Feed, Low-Profile, Multi-Layered, Circularly Polarized Patch Antenna
}

\author{
Ming-Chun Tang, Senior Member, IEEE, Xiaoming Chen, Student Member, IEEE, Mei Li, Member, \\ IEEE, and Richard W. Ziolkowski, Fellow, IEEE
}

\begin{abstract}
A bandwidth-enhanced, compact, low profile, single-feed, multi-layered, circularly polarized (CP) patch antenna is presented. A corner-truncated patch is introduced as a near-field resonant parasitic (NFRP) element directly beneath a specially-engineered radiation patch. Without sacrificing the antenna's low profile and compact size, its presence not only introduces a new pair of orthogonal near-degenerate resonant modes, but it also recovers a similar pair from the cross-slots of the main patch. With the aid of both circular slots and meander-line slots on these patches, the resulting three pairs of adjacent near-degenerate modes have been successfully combined with the same clockwise polarization to enhance the CP bandwidth by more than a factor of two when compared with the same-height conventional single-layer patch antennas. Measured results are in good agreement with their simulated values and demonstrate that the reported antenna is low profile: $0.016 \lambda_{0}$, achieves a -10dB impedance bandwidth of $\sim 4.6 \%$, and a 3-dB AR bandwidth of about $2.33 \%$ along with realized gains of $4.5 \pm 0.15$ $\mathrm{dBi}$, throughout that bandwidth. Analyses of the current distributions are used to explain the contributions of the parasitic patch and further simulation studies validate our design guidelines and show its advantages.
\end{abstract}

Index Terms-Bandwidth, circular polarization, compact antenna, low-profile antenna, patch antenna

\section{INTRODUCTION}

$\mathrm{C}$ ompared with dual and other multi-feed versions, the single-feed CP patch antennas are valued because their configurations are inherently simpler. Nevertheless, most reported single-feed CP patch antennas suffer from an inherent,

Manuscript received on Apr. 05, 2017, revised on May 19, 2017, and accepted on 04 June, 2017.

This work was supported in part by the National Natural Science Foundation of China contract number 61471072, in part by graduate scientific research and innovation foundation of Chongqing, China contract number CYS16020, in part by Chongqing Postdoctoral Special Funding Project contract number xm2016022, in part by the Funding of the Young Backbone Teachers in Colleges and Universities of Chongqing contract number 0307001104102, and in part by the Australian Research Council grant number DP160102219.

M. -C. Tang, X. Chen, and M. Li are with the Key Laboratory of Dependable Service Computing in Cyber Physical Society Ministry of Education, College of Communication Engineering, Chongqing University (tangmingchunuestc@126.com);

R. W. Ziolkowski is with the University of Technology Sydney, Global Big Data Technologies Centre, Ultimo NSW 2007, Australia, and the Department of Electrical and Computer Engineering, University of Arizona, Tucson, AZ 85721, USA (E-mail: richard.ziolkowski@uts.edu.au). very narrow axial ratio (AR) bandwidth. This aspect severely limits their widespread application [1-4]. A popular and effective method to improve their AR bandwidths is to introduce additional parasitic patches [5-14]. Unfortunately, their presence also has some inescapable drawbacks. For instance, vertically stacking one or more parasitic patches with similar configurations has the desired effects, but it also significantly increases the antenna's total height [5-13]. On the other hand, adding parasitic patches around the main radiating patch on the same layer requires much more area than the main patch $[13,14]$.

In this letter, a single-feed, square corner-truncated, near-field resonant parasitic (NFRP) patch is placed directly beneath a specially-engineered radiation patch to improve the AR bandwidth, while achieving very good impedance matching and maintaining its low profile. It is demonstrated that the presence of this NFRP patch not only introduces a new pair of orthogonal near-degenerate resonant modes, but it also recovers a similar pair from the cross-slots introduced into the radiating patch. At the same time it improves the performance of the original pair of near-degenerate modes from the corner-cuts of that patch. It will be shown that by introducing both circular slots and meander-line slots on these patches, three pairs of adjacent near-degenerate modes with the same clockwise polarization successfully combine to produce twice the CP bandwidth when compared to conventional patch antennas of the same height [5-14] without sacrificing the compact size advantages. Simulation results are compared with the measurements of a fabricated prototype; very good agreement is demonstrated. The measured results show that the reported antenna is low profile: $0.016 \lambda_{0}$ ( $\lambda_{0}$ being the wavelength corresponding to its centered operating frequency); achieves a $4.6 \%$ impedance bandwidth for $\left|\mathrm{S}_{11}\right|<-10 \mathrm{~dB}$ and a 2.33\% 3-dB AR bandwidth; and produces a realized gain level over $4.3 \mathrm{dBi}$ within that 3-dB AR bandwidth. Analyses of the current distributions and design process are used to explain the contributions of the parasitic patch.

Note that all of the numerical simulations and their optimizations were performed using the frequency domain ANSYS/ANSOFT High Frequency Structure Simulator (HFSS), version 13.0 [15].

\section{Antenna Design}

The geometry of the proposed antenna is illustrated in Fig. 1. The optimized design parameters are given in Table I. This 
antenna was designed for fabrication with printed circuit board (PCB) technology. Two pieces of planar copper-cladded Rogers 4350B substrates whose relative dielectric constant $\varepsilon_{r}=$ 3.48, loss tangent $\tan \delta=0.0038$, and $0.5 \mathrm{oz}(17 \mu \mathrm{m})$ thick copper film were utilized. These two components have the same dimensions: $L_{g} \times W_{g} \times h_{0}=100 \mathrm{~mm} \times 100 \mathrm{~mm} \times 1.5 \mathrm{~mm}$, and are shown in Fig.1 (a). The design is simple and yields a compact configuration.

As is shown in Fig. 1(b), a specially-engineered patch serves as the main radiator. Its transverse size is $L_{1} \times W_{1}$. It is etched on the top of the upper substrate piece and is connected directly and perpendicularly to the center probe of an SMA connector (whose radius is $0.6 \mathrm{~mm}$ ). Two same-sized corner cuts lie along one diagonal. They have the ability to excite a pair of two near-degenerate orthogonal modes which give rise to one $\mathrm{CP}$ radiation mode [1, 2]. Additionally, a cross slot is also etched in the center of this patch to achieve another CP mode [16]. However, without any further modifications, only one CP radiation mode can be excited for such a coax-fed design, even though both features exist in the same radiating patch. This phenomenon will be illustrated in Section IV. Moreover, three slots are cut from opposite edges of the patch along the $y$-axis to form two meander-line features. Their presence allows one to reduce the size of the patch and to adjust the resulting AR values. Furthermore, two additional circular slots with radius $R_{1}$ are etched along the other diagonal of this top patch to further optimize its CP performance.

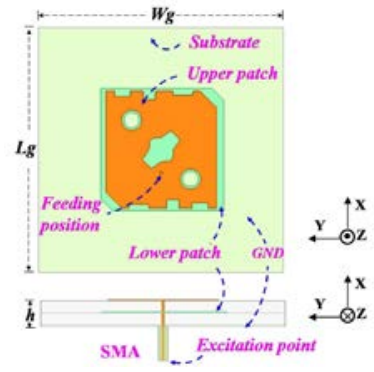

(a)

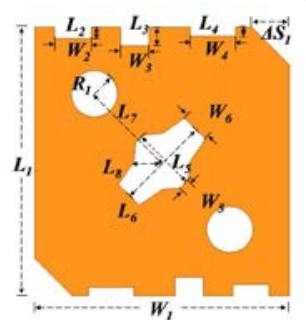

(b)

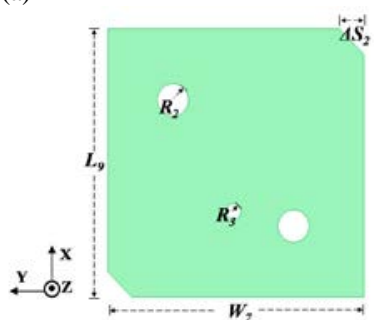

(c)
Fig. 1 The geometry of the proposed antenna. (a) Top and side view of the whole antenna, (b) top view of the upper (radiating) patch, and (c) top view of the lower (NFRP) patch.

The second patch is shown in Fig. 1(c). It is etched directly under the top patch on the lower face of the top substrate piece. It has a different size: $L_{9} \times W_{7}$, but it also has a configuration similar to the main patch. It serves as a NFRP element [17]. While it also has two same-sized corner cuts along the same diagonal as the radiating patch, it introduces another $\mathrm{CP}$ mode because of its slightly different size. In addition, two circular slots are etched with radius $R_{2}$ from this NFRP patch directly beneath the slots on the upper face and centered on them. They allow further adjustments of the overall CP performance.
Finally, yet another circular slot is etched on the NFRP patch with a radius $R_{3}$ which is large enough so that the SMA probe will pass through it without any contact.

The bottom substrate piece is the largest component. It is square with size: $L_{g} \times W_{g}$. Its bottom face is entirely copper; it acts as the ground plane for this antenna. The copper was removed from its top face. The bottom face has a circular slot etched from it whose radius $R=2 \mathrm{~mm}$ is identical to the radius of the outer conductor of the coax.

TABle I. The Optimized Design PARAMETERS OF THE SingLE-FEEd CP PATCH ANTENNA (ALL DIMENSIONS ARE IN MILLIMETERS)

\begin{tabular}{|c|c|c|c|}
\hline$L_{g}=100$ & $L_{1}=47.5$ & $L_{2}=2$ & $L_{3}=3.3$ \\
\hline$L_{4}=1.5$ & $L_{5}=11.2$ & $L_{6}=16.6$ & $L_{7}=12$ \\
\hline$L_{8}=3.5$ & $L_{9}=47.65$ & $W_{g}=100$ & $W_{1}=45.1$ \\
\hline$W_{2}=6.4$ & $W_{3}=5$ & $W_{4}=8$ & $W_{5}=2$ \\
\hline$W_{6}=5.2$ & $W_{7}=47.65$ & $R_{1}=4.1$ & $R_{2}=3.1$ \\
\hline$R_{3}=1.5$ & $\Delta S_{1}=6.6$ & $\Delta S_{2}=4.8$ & $h=3$ \\
\hline
\end{tabular}

\section{Simulated AND MEASURED RESUlts}

The radiation performance characteristics of the proposed antenna were investigated numerically and experimentally. As shown in Fig. 2, the components of a prototype of the optimized antenna were fabricated and assembled. The reflection coefficients are presented as a function of the excitation frequency, together with their simulated values, in Fig. 2(a). It is clear that the presence of the NFRP element produces several resonant modes, which in turn produce an improvement of the impedance bandwidth. In particular, the simulated $-10 \mathrm{~dB}$ bandwidth was from 1.578 to $1.645 \mathrm{GHz}(\sim 4.1 \%$ fractional bandwidth), while the measured bandwidth was from 1.566 to $1.639 \mathrm{GHz}$ ( 4.6\% fractional bandwidth). The measured center of the operational frequencies witnessed only a $9.0 \mathrm{MHz}$ red-shift $(\sim 0.56 \%)$ from its simulated value.

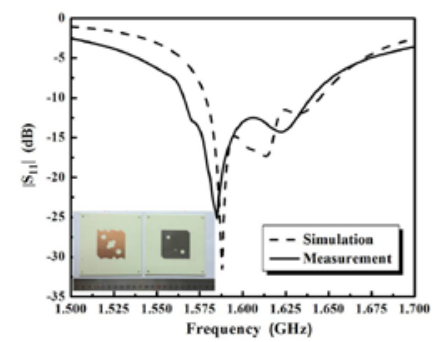

(a)

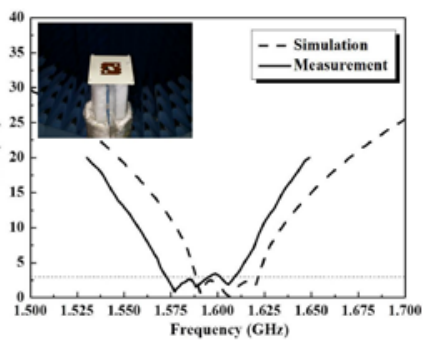

(b)

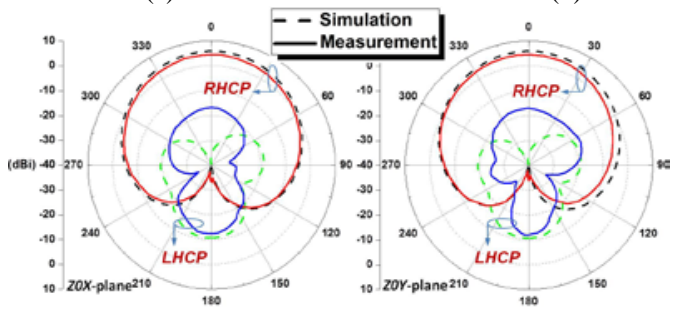

(c)

Fig. 2 Measured and simulated performance characteristics of the CP patch antenna. (a) $\left|S_{11}\right|$ and (b) AR values as functions of the source frequency; and (c) the $2 \mathrm{D}$ radiation patterns at the measured (simulated) center frequency point 1.606 (1.588) GHz within the 3dB AR bandwidth.

The pattern and AR measurements were carried out in an anechoic chamber at the University of Electronic Science and Technology of China (UESTC). The measured and simulated AR values at boresight are shown in Fig. 2(b) as functions of 
the source frequency. It is readily seen that the measured (simulated) AR values are lower than $3 \mathrm{~dB}$ in the frequency range from 1.572 (1.588) to 1.609 (1.621) GHz. Thus, the antenna has a measured (simulated) 37 (33) $\mathrm{MHz}$ AR bandwidth, which is approximately a 2.33 (2.08) \% fractional AR bandwidth. This AR bandwidth is enhanced by more than a factor of two when compared to the value obtained from a same sized conventional patch antenna. Within this range, three adjacent CP radiation modes can be easily observed at $1.577 \mathrm{GHz}(1.591 \mathrm{GHz}), 1.588 \mathrm{GHz}(1.606 \mathrm{GHz})$ and $1.606 \mathrm{GHz}$ $(1.619 \mathrm{GHz})$, respectively. Moreover, the $3 \mathrm{~dB}$ AR beamwidths are stable and cover more than $-60^{\circ}$ to $+60^{\circ}$ over its $3 \mathrm{~dB}$ AR bandwidth. Note that the measured AR results are slightly above $3 \mathrm{~dB}$ in a very small region around $1.599 \mathrm{GHz}$. Also note that when the measured AR values are larger than $20 \mathrm{~dB}$, the SATIMO system software deems them to be equal to $20 \mathrm{~dB}$ and, thus, they are not plotted in Fig. 2(b). The measured and simulated radiation patterns at the center minimum AR value frequency point are presented in Fig. 2(c). It is observed that the antenna operates in right-handed CP (RHCP) with stable radiation patterns.

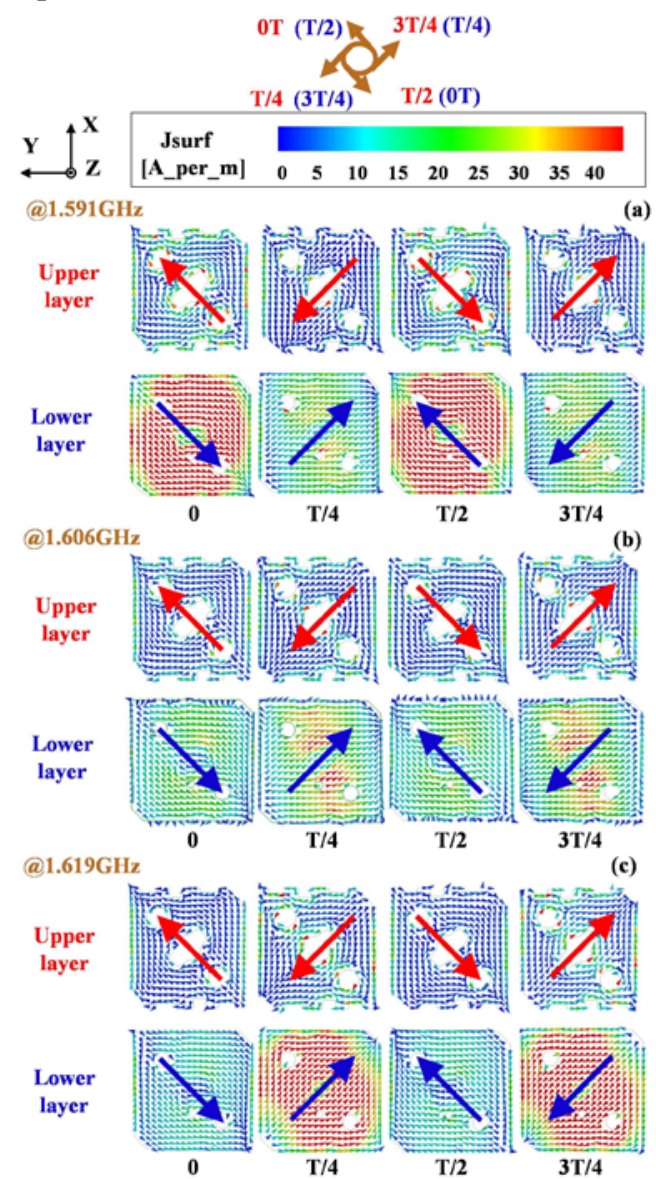

Fig. 3 The current distributions on the upper surfaces of the two patches over one period, $T=1 / f_{0}$, corresponding to the three minimum AR value frequency points: $f_{0}=$ (a) 1.591 , (b) 1.606 , and (c) $1.619 \mathrm{GHz}$.

The measured (simulated) boresight realized gains are, respectively, $4.63(4.170) \mathrm{dBi}$, $4.51(5.84) \mathrm{dBi}$, and 4.37 (4.40) $\mathrm{dBi}$, at the three $\mathrm{AR}_{\min }$ frequency points: $1.591(1.577) \mathrm{GHz}$, 1.606 (1.588) GHz, and 1.619 (1.606) GHz. Moreover, the measured (simulated) half-power beamwidths are approximately $87^{\circ}$ over the entire operational $3 \mathrm{~dB}$ AR bandwidth. These values demonstrate that the measured $\mathrm{CP}$ prototype antenna radiates efficiently with a stable gain performance.

\section{OPERATIONAL MeCHANISMS}

To explain the operational mechanisms of this CP antenna, the current distributions on the upper surfaces of its top patch and NFRP patch were investigated. They are illustrated in Fig. 3 over a period corresponding to the frequency of each of the three minimum AR values. The effective directions of these surface currents are highlighted with additional red (referring to the top patch) and blue (referring to the NFRP patch) arrows. It is clear at each frequency point that the surface currents on both patches exhibit the same RHCP performance, but are $180^{\circ}$ out of phase with each other.

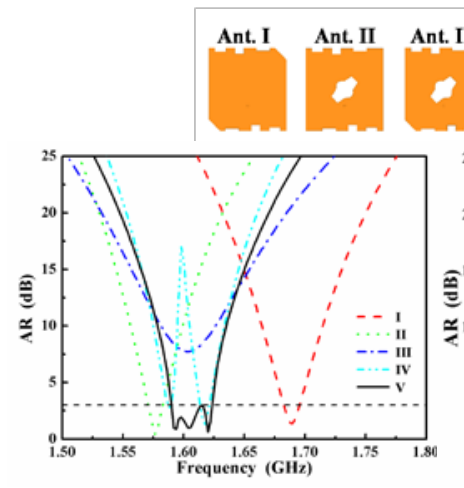

(a)
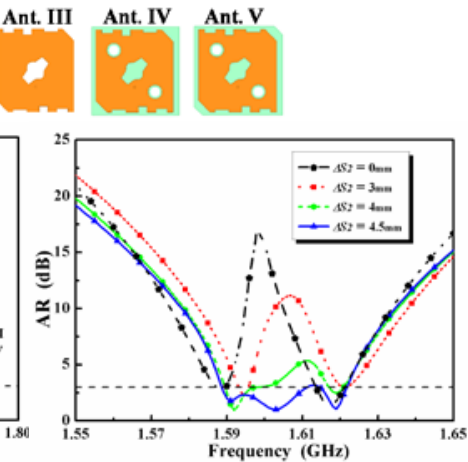

(b)
Fig. 4 Cases studied to explain the CP bandwidth enhancement. (a) The AR values of each design as it evolves from Ant. I to Ant. V. (b) As the length, $\Delta S_{2}$, of the corner cuts of the NFRP patch in Ant. V is increased, the associated AR values confirm the appearance of the third CP mode.

To understand how the NFRP patch improves the CP bandwidth of the system, the AR values as functions of the source frequency are compared in Fig. 4 for several different designs. Ant. I and Ant. II are single-layer square patch antennas, respectively, with an etched pair of corner cuts and one cross slot. As Fig. 4(a) indicates, both are large enough to obtain $\mathrm{CP}$ radiation ( $\mathrm{AR} \leq 3 \mathrm{~dB}$ ) from two near-degenerate orthogonal modes that produce electric field components of comparable magnitudes and quadrature phase difference [1, 2]. However, when both the cross slot and the corner cuts are etched directly on the same patch (Ant. III), one cannot take advantage of both effects simultaneously to generate two independent $\mathrm{CP}$ modes. Their mutual presence prevents the requisite currents from forming. As a result, only a single $\mathrm{CP}$ radiation mode is observed in Fig. 4(a).

In order to obtain the desired simultaneous presence of two or more $\mathrm{CP}$ radiation modes on the radiating patch and, hence, to enhance its AR bandwidth, one has to introduce a second structure that can be tailored to control the associated current flows. The NFRP patch plays this role. A dual-layer version with a simple square NFRP patch, Ant. IV, confirms that its presence does redistribute the current paths on the radiating patch to facilitate the re-appearance of the ghost CP mode. As its AR values show in Fig. 4(a), Ant. IV has two CP radiation modes; they are centered at 1.588 and $1.618 \mathrm{GHz}$. The mutual capacitance between both patches allows further tuning of its resonance frequencies [16]. 
Parameter studies show that when the corner-cut lengths, $\Delta S_{1}$, in the upper patch of Ant. IV are increased, the higher $A R_{\text {min }}$ frequency exhibits a blue-shift, while the lower $A R_{\text {min }}$ frequency remains unchanged. On the other hand, when only the total cross slot size $L_{6}$ is increased, the lower $A_{\text {min }}$ frequency experiences a red-shift, while the higher $\mathrm{AR}_{\text {min }}$ frequency remains the same. Finally, to further tune those resonance frequencies and to introduce yet another CP radiation mode between them, additional perturbations - two corner cuts - are introduced on the NFRP patch of Ant. V. Fig. 4(b) shows that as the length of those corner cuts, $\Delta S_{2}$, is increased, the AR values of Ant. IV are transformed into those of the optimized Ant. V design. The third mode appears between the other two when the cuts are large enough; its $\mathrm{AR}_{\text {min }}$ value is then tailored with further fine tuning to yield the desired third CP mode. As Figs. 4(a) and 4(b) summarize, the overlap of all three adjacent $\mathrm{CP}$ radiation modes produces the measured (simulated) enhanced CP bandwidth.

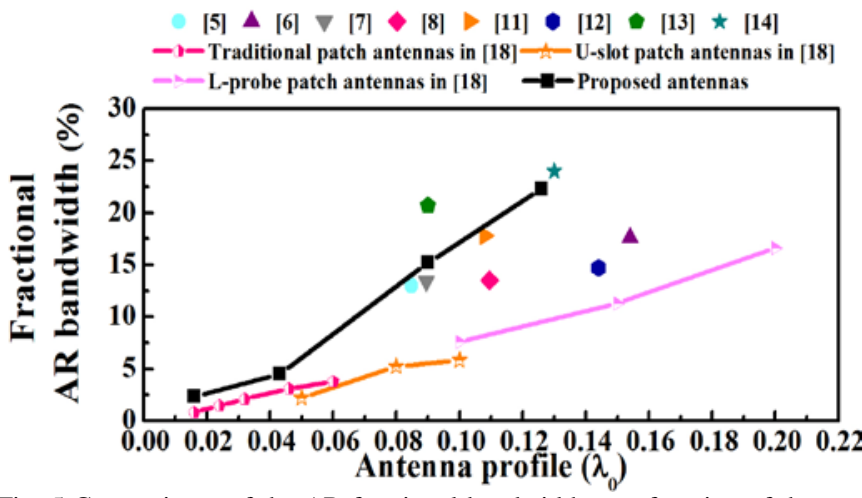

Fig. 5 Comparisons of the AR fractional bandwidth as a function of the total antenna profile (height) for our NFRP CP antennas and similar single-feed CP antennas reported in the literature.

As noted initially, our NFRP CP design strategy not only improves the AR bandwidth, but also maintains the total height and compact size of the resulting antennas. When these characteristics are compared to those of the many single-fed CP antennas reported in the literature, our designs offer distinct advantages, particularly with their enhanced AR bandwidths. Fig. 5 is provided to emphasize these comparisons of antenna height and AR bandwidth in a comprehensive manner. Several additional antenna-height designs are included that were obtained and optimized with our strategy in order to make these comparisons fair. It is clearly observed that our antennas have wider AR bandwidths than most of the referenced comparison antennas of the same height $[5-12,18]$. One exception is the approach in which parasitic patches are added around the main radiating patch on the same layer $[13,14]$. Nevertheless, with these additional radiating elements, the entire area of those systems is five-times larger than ours. Furthermore, the curves in Fig.5 indicate that our design strategy is much more suitable in general to obtain AR bandwidth enhancement for ultra-low profile CP antennas, especially when the total height $\mathrm{h}<0.05 \lambda_{0}$.

\section{CONCLUSIONS}

A compact, bandwidth-enhanced, low profile, single-feed, multi-layered, CP patch antenna was reported. The main attributes of the design were the presence of meander-line and circular slot features on the corner-cut and slot modified top patch and the introduction of a NFRP patch modified with corner-cuts and circular slots. The introduction of all of these features led to an AR bandwidth more than twice as large as similar sized conventional patch antenna. The antenna's low profile, $0.016 \lambda_{0}$, and compact physical size, were not sacrificed to achieve this enhancement. Measured results, in good agreement with their simulated values, indicate that the reported antenna achieved a $4.6 \%$ fractional $-10 \mathrm{~dB}$ impedance bandwidth, a $2.33 \% 3 \mathrm{~dB}$ AR bandwidth, and produces a realized gain level over $4.3 \mathrm{dBi}$ within that $3-\mathrm{dB}$ AR bandwidth. The antenna itself could have future global positioning system (GPS) applications. Moreover, the presented design principals can lead to improvements in current CP patch antennas and those being considered for future wireless applications.

\section{REFERENCES}

[1] C. A. Balanis, Antenna Theory: Analysis and Design. New York: Wiley, 2005.

[2] S. Gao, Q. Luo, and F. Zhu, Circularly Polarized Antennas. West Sussex: Wiley-IEEE Press, 2014.

[3] W.-S. Chen, K.-L. Wong, and C.-K. Wu, "Inset microstripline-fed circularly polarized microstrip antennas," IEEE Trans. Antennas Propag., vol. 48, no. 8, pp. 1253-1254, Aug. 2000.

[4] Nasimuddin, Z. N. Chen, and X. Qing "Asymmetric-circular shaped slotted microstrip antennas for circular polarization and RFID applications," IEEE Trans. Antennas Propag., vol.58, no. 12, pp. 3821-3828, Dec. 2010.

[5] N. Herscovici, Z. Sipus, and D. Bonefačić, "Circularly polarized single-fed wide-band microstrip patch," IEEE Trans. Antennas Propag., vol. 51, no. 6, pp. 1277-1280, Jun. 2003.

[6] Nasimuddin, and K.P. Esselle "New feed system for wideband circularly polarised stacked microstrip antennas," IET Microw. Antennas Propag., vol. 1, no. 5, pp. 1086-1091, 2007.

[7] Nasimuddin, X. Qing, and Z. N. Chen, "A wideband circularly polarized stacked slotted microstrip patch antennas," IEEE Antennas Propag. Mag., vol. 55, no. 6, pp. 84-99, Dec. 2012.

[8] Z. B. Wang, S. J. Fang, S. Q. Fu, and M. Fan, "Single-fed broadband circularly polarized stacked patch antenna with horizontally meandered strip for universal UHF RFID applications," IEEE Trans. Microw. Theory Tech., vol. 59, no. 4, pp. 1066-1073, Apr. 2011.

[9] H. Oraizi and R. Pazoki, "Wideband circularly polarized aperture-fed rotated stacked patch antenna," IEEE Trans. Antennas Propag., vol. 61, no. 3, pp. 1048-1054, Mar. 2013.

[10] X. Ta and I. Park, "Low-profile broadband circularly polarized patch antenna using metasurface," IEEE Trans. Antennas Propag., vol. 63, no. 12, pp. 5929-5934, Dec. 2015.

[11] Nasimuddin, Z. N. Chen, and K. P. Esselle, "Wideband circularly polarized microstrip antenna array using a new single feed network," Microw. Opt. Technol. Lett., vol. 50, no. 7, pp. 1784-1789, 2008.

[12] Nasimuddin, K. P. Esselle, and A. K. Verma, "Wideband circularly polarized stacked microstrip antennas," IEEE Antennas and Wireless Propag. Lett., vol. 6, pp. 21-24, 2007.

[13] W. Yang, J. Zhou, Z. Yu, and L. Li, "Single-fed low profile broadband circularly polarized stacked patch antenna," IEEE Trans. Antennas Propag., vol. 62, no. 10, pp. 5406-5410, Oct. 2014.

[14] J. Wu, Y. Yin, Z. Wang, and R. Lian, "Broadband circularly polarized patch antenna with parasitic strips," IEEE Antennas Wireless Propag. Lett., vol. 14, pp. 559-562, 2015.

[15] ANSYS/ANSOFT High Frequency Structure Simulation (HFSS), ver. 13.0, ANSYS Corp. [Online]. Available at www.ansoft.com.

[16] J.-M. Chen and J.-S. Row, "Wideband circularly polarized slotted-patch antenna with a reflector," IEEE Antennas Wireless Propag. Lett., vol. 14, pp. 575-578, 2015.

[17] R. W. Ziolkowski, P. Jin, and C.-C. Lin, "Metamaterial-inspired engineering of antennas," Proc. IEEE, vol. 99, no. 10, pp. 1720-1731, Oct. 2011.

[18] S. S. Yang, K.-F. Lee, and A. A. Kishk, "Design and study of wideband single feed circularly polarized microstrip antennas," Prog. Electromagn. Res., vol. 80, pp. 45-61, 2008. 